\title{
Therapeutic potential of totipotent, pluripotent and multipotent stem cells
}

\begin{abstract}
The discovery of stem cells opened up a whole new arena of research for scientists. And the realizations of their therapeutic potential, promises sustainable cure for previously untreated diseases. Stem cells may broadly be classified into totipotent, pluripotent and multipotent and each of these classes show enormous therapeutic function. Totipotent stem cells can differentiate into 200 cell types of the body and also has the ability to reconstitute a stem cell-deprived organ, thereby opening ways to tissue regeneration and replacement therapies respectively. Similarly, Pluripotent stem cells have been made to differentiate into neural tissues, insulin secreting cells, cardiomyocytes, hematopoietic cells, osteoblasts, endothelial cells and hepatocytes successfully. Also, the prospects for reprogramming human somatic cells into induced Pluripotent Stem (iPS) cells have heralded a new era in the field of Stem Cell Therapeutics. In contrast, Multipotent stem cells also promises self-renewal as well as demonstrates plasticity to transdifferentiate into muscle, skeletal, liver, kidney, muscle, skin, neural, and cardiac cell lineages. Additionally, they are shown to serve as a gene delivery vehicle in vivo. This review spans around the therapeutic applications of these three major types of stem cells.
\end{abstract}

Keywords: totipotent stem cells, pluripotent stem cells, multipotent stem cells, induced pluripotent stem cells, transdifferentiation, endothelial progenitor cells, myeloid lineage, lymphoid lineage
Volume 2 Issue 5 - 2015

\author{
Kiran Makhani,' Salima Mehboob Ali,' Sarah \\ Yousuf,' Shezaib Siddiqui ${ }^{2}$ \\ 'Department of Biotechnology, University of Karachi, Pakistan \\ 2International Center for Chemical and Biological Sciences \\ (ICCBS), University of Karachi, Pakistan
}

\begin{abstract}
Correspondence: Shezaib Siddiqui, Dr. Panjwani Center for Molecular Medicine and Drug Research (PCMD), International Center for Chemical and Biological Sciences (ICCBS), University of Karachi, Pakistan, Tel 923353683716,
\end{abstract} Email shehzaib_siddiqui@hotmail.com

Received: November 21, 2015 | Published: December 17, 2015
Abbreviations: MHC, major histocompatibility complex; IPS, induced pluripotent stem cells; MSCs, mesenchymal stem cells; EPCs, endothelial progenitor cells; BM, bone marrow; hTERT, human telomerase reverse transcriptase

\section{Introduction}

The two fundamental characteristics of stem cells are the ability of unlimited self-regeneration and the production of at least one type of differentiated progeny cells. ${ }^{1}$ Different kinds of stem cells have different potentials of differentiation. Totipotent stem cells are able to produce all types of cells. A zygote is a totipotent stem cell which can even produce cells that are not actually the part of the embryo, example placental cells. During embryonic development, cells gradually become confined to distinct paths of differentiation and hence become pluripotent that is they lose the capacity of forming extra embryonic tissue but retains the ability of differentiating into all types of adult cells. ${ }^{2}$ As the development continues, cells, previously pluripotent become restricted hence now called as multipotent. Distinct characteristic of multipotent stem cells is they can only differentiate into cells of a particular lineage. For example hematopoietic stem cells possess the ability to produce all kinds of blood cells but not neurons or lung cells. ${ }^{3}$ The continuous self-renewal and differentiating ability of stem cells has created modern research emphasis on it, especially in relation to its therapeutic use. ${ }^{2}$

\section{Therapeutic potential of totipotent stem cells}

Totipotent stem cells: Totipotent stem cells, however, out of all the other types, presents an ideal approach to deal with the therapies related to the diseases. ${ }^{4}$ Totipotency- a potential exhibited by the cell upon fusion of an egg with a sperm characterizes it among the most versatile class of cells. ${ }^{5}$ The zygote that develops as a result of fusion of the gametes is a single totipotent cell which has the capability to divide into numerous identical cells; giving rise to any of the three germ layers of human (endoderm, ectoderm or mesoderm) as well as extra embryonic cells which include cytotrophoblast layer and a syncytiotrophoblast layer of the placenta. ${ }^{6}$ In addition, the feature which makes these cells so distinctive from the others is the potential to proliferate and differentiate into a complete organism. ${ }^{4}$ Unlike pluripotent cells which can specialize to form only the embryonic cells ${ }^{7}$ and multipotent cells which can give rise to only two or more mature cell types, ${ }^{8}$ totipotent cells can replicate unlimitedly and differentiate into nearly all human cells such as of nerve and heart. ${ }^{5}$ Correspondingly, the totipotent cells do not just have the potential to replicate into 16 identical cells within 4 days but they do so without even losing their Totipotency. When the zygote replicates to reach a 16-celled stage, it, however, starts to lose its Totipotency and transits to differentiate into a specialized cell. ${ }^{6}$

Differentiating and regenerative potential: In accordance with studies and the experiments that have been conducted so far, the potency of the totipotent cells cannot be undermined by the fact that it can differentiate itself into about 200 cell types of a human body. ${ }^{6}$ Several experiments have been carried out to observe this potential which includes the pioneering efforts made on rats as well as some on mammalian species by numerous scientists. ${ }^{9-12}$

In 1992, results were documented that totipotent hematopoietic stem cells pretreated with 5-fluorouracil when injected into the mouse were observed to differentiate into myeloid and lymphoid progeny with a long term in vivo reconstituting ability even after 7 months of transplantation..$^{13}$ Similarly, another research conducted in the earlier years also reported the same result of differentiation of totipotent 
hematopoietic stem cells into myeloid and lymphoid lineages when seeded into the mouse with mild and severe endogenous defects. ${ }^{14}$ Also, the research conducted using the totipotent cells and inducing them to differentiate into a variety of cells when cultured as embryonic bodies along with the adult neural stem cells in order to induce differentiation, has resulted in the generation of myocytes- the cells found in muscle tissue. ${ }^{15,16}$

Therefore, successful differentiation and enormous therapeutic potentials of the totipotent cells has led scientists to envision the possible treatments of diseases through cell and gene therapy as well as tissue regeneration. ${ }^{5}$ The replacement therapy whereby the totipotent stem cells are differentiated into the desired cells and subsequently transplanted into the damaged tissue has proved to be one of the ideal approaches in restoring the functions of the tissue. ${ }^{17}$ Moreover, stem cell based treatments via regenerative strategy wherein the abnormal cells are replaced by the differentiated stem cells and tropic strategy which involves the secretion of several different mediators such as interleukins and neurotrophic factors has also enabled scientist to cure diseases, particularly retinal associated diseases. ${ }^{18}$

\section{Therapeutic potentials of pluripotent stem cells}

Replacing the pluripotent stem cells: Several diseases are characterized by exhaustion of stem cells in body such as Fanconi Anemia (Genetic defects in hematopoietic stem cells) or failure of bone marrow due hematopoietic stem cells which results in leukemia and lymphoma. Other diseases like type 1 diabetes due to auto immune destruction of pancreatic beta cells and liver cirrhosis generate a condition in which cells of the affected organ cannot be replenished actively from stem cells of the body. Such diseases can be treated by replacing the stem cell pool or with direct organ transplantation. The lack of healthy and matched donors has made the method of using pluripotent stem cells more ideal. ${ }^{19}$ Another report showed that the presence of hoxB4 gene stimulates the production of hematopoietic stem cells in mice, henceforth promising a therapy for humans with impaired hematopoiesis. ${ }^{20}$

Differentiation and therapeutic application: In several trials, mouse pluripotent stem cells derived dopaminergic neurons have shown to repair motor dysfunction of Parkinson cases in rodent models. ${ }^{21,22}$ Human pluripotent stem cells have also been differentiated into pancreatic cells producing insulin which can play a significant role for treating type I diabetes. Somatostatin and glucagon secreting pancreatic cells have also produced. ${ }^{23}$ Aging and accidental injuries can cause irreversible destruction of cardiac tissues due to their rare mitosis characteristic which restricts the natural regeneration of myocardial cells. Successful attempts have been made to use pluripotent stem cells for cardiac grafting. ${ }^{24,25}$ Trials for whole blood formation have also being done in mouse models. ${ }^{26}$ However, obtaining stable blood derived from pluripotent embryonic stem cells was challenging. ${ }^{27}$ Using human pluripotent stem cells neural tissues, insulin secreting cells, cardiomyocytes, hematopoietic cells, osteoblasts, endothelial cells and hepatocytes are being successfully produced. ${ }^{19}$

\section{Limitations}

As inevitable, among all types of stem cells pluripotent stem cells have invaluable potential for therapeutic purposes but the problem of immune rejection of transplanted cells is a major hindrance in its wide spread clinical use. For example, embryonic pluripotent stem cells derived hematopoietic cells express a low level of class I MHC and hence become a target of natural killer cells. Along with this, the use of human embryos has become a major social and religious concern. ${ }^{28,29}$

\section{Induced pluripotent stem cells}

In consideration to such problems, recently, trials for reprogramming human somatic cells into induced pluripotent stem cells are being performed using Oct4, Sox2, Klf4 and Myc transcription factors. ${ }^{30}$ Induced pluripotent stem cells hold great promise for drug discovery, understanding disease mechanisms, cells and tissue replacement therapies and regenerative medicine. ${ }^{29}$

Reprogramming of anemic mouse fibroblast cells and correction of gene by homologous recombination led to successful differentiation of the resulting iPS cells into hematopoietic progenitor cells which were subsequently transplanted back in to the mouse. Visible recovery was observed in the mouse after transplantation. ${ }^{30}$ Dopamine neurons derived from iPS cells that were transplanted into adult rat brain suffering with Parkinson disease proved to provide substantial recovery from the diseased phenotype.$^{31}$ Ebert $\mathrm{AD}$ et al. ${ }^{32}$ reports the generation of motor neurons using iPS cells derived from somatic cells of a patient diagnosed with spinal muscular atrophy. ${ }^{32}$ Mice suffering with hemophilia A were protected in a death inducing bleeding assay after injection of iPS cells derived endothelial cells into the liver. ${ }^{33}$ As the production of iPS cells involves the reprogramming of somatic cells therefore the use of iPS cells in the treatment of epigenetic related defects like myelodysplastic syndrome is valuable and possesses the potential to completely reverse the disease condition. ${ }^{34}$ Robust advancements in iPS cells production have also led to the generation of clinically relevant cell lines free of genomic integration and oncogenes which will ultimately contributes to the development of several therapies for genetic and epigenetic defects. ${ }^{29}$

\section{Therapeutic potential of multipotent stem cells}

Multipotent stem cells are cells that have the capacity to selfrenew by dividing and to develop into multiple specialized cell types present in a specific tissue or organ. ${ }^{35}$

Self-renewal: Mesenchymal stem cells (MSCs) found in many adult tissues are an attractive multipotent stem cell source. ${ }^{36}$ During the last two decades, mesenchymal stem cells (MSCs) have emerged as a potential player for cell-based therapeutics. They have demonstrated efficacy in multiple types of cellular therapeutic strategies, including applications in treating children with osteogenesis imperfect, hematopoietic recovery, and bone tissue regeneration strategies. ${ }^{37-39}$ Moreover, the ability of MSCs to differentiate into a variety of connective tissue cell types has rendered them useful in the augmentation, local repair and regeneration of bone, cartilage and tendon. ${ }^{40-43}$ MSCs are also located in the wall of large and small blood vessels in, but not limited to, brain, spleen, liver, kidney, lung, muscle, thymus, and pancreas. ${ }^{44,45}$ Therefore, they may serve as a cell source for therapies of diverse disorders such as diabetes, osteoporosis, arthritis, muscular degenerative disorders, and the regeneration blood vessel walls after tissue injuries. ${ }^{46-49}$

Human studies have reported autologous whole bone marrow to improve posterior spinal fusion in adolescent idiopathic scoliosis when administered along with demineralize bone matrix..$^{50}$ Similar human trials have reported the efficacy of using autologous bone marrow aspirates combined with mineralized collagen matrix grafts for fusion of thoracolumbar fractures. ${ }^{51}$ 
In addition to the MSCs, Hematopoietic Stem Cells may be used in autologous or allogeneic transplantations for the treatment of patients with diverse hematopoietic disorders and several inherited immune-deficient and autoimmune diseases and to reconstitute the hematopoietic cell lineages and immune system defense..$^{52,53}$

The in vivo induction of mobilization of BM-derived Endothelial Progenitor Cells (EPCs) into peripheral circulation or their activation can open prospects to promote neo-angiogenesis and vascular repair of injured areas. ${ }^{45,53-57}$ As evidence, it has been observed that the injection of isolated human CD34-/CD133+/VEGFR2+/ CD14EPC sub-population in nude mice with an injury in carotid artery led to their differentiation into endothelial cells and their subsequent incorporation into the endothelial layer of carotid artery lesion, resulting in a decrease in lesion size..$^{55}$

NSCs, genetically modified NSCs, or their further differentiated progeny could be used as therapeutic agents, owing to their intrinsic capacity to restore dysfunctional neurons in the brain by releasing neuro-regenerative molecules. ${ }^{58}$ They are proposed to treat a variety of central nervous system disorders and several progressive and neurodegenerative diseases, characterized by deterioration and/or loss of neuronal cells, like, multiple sclerosis, Parkinson's, Alzheimer's, Amyotrophic Lateral Sclerosis, and Huntington's diseases. .3,59-64 $^{-6}$ Moreover, this restoration of lost neuronal cells could also improve impairment in memory loss, abnormal control of movement, sensation, and other autonomic nervous functions.

Trans-differentiation: Recent advancements suggest that adult multipotent stem cells, previously thought to undergo tissue-specific differentiation, are potent of trans-differentiation as well. Transdifferentiation refers to differentiation of a cell into lineages other than its tissue of origin. For instance, bone marrow (BM) cells are observed to possess the ability to repopulate many non-hematopoietic tissues, such as neuro-ectodermal cells, skeletal myoblasts, cardiomyocytes, endothelium, hepatocytes and lung, gut, and skin epithelia. ${ }^{65-72}$ Whereas, BM-derived circulating Hematopoietic Stem Cells and Endothelial Progenitor Cells within peripheral circulation are able to Trans-differentiate into cells of damaged non-hematopoietic tissues. ${ }^{73}$ Also, BM-derived stem cells, such as endothelial progenitor cells, angioblasts, or CD34+ cells, when transplanted into ischemic myocardium incorporate into the sites of neovascularization and have shown to improve cardiac function. ${ }^{74-76}$ Another evidence of trans-differentiation of multipotent stem cells reside in the ability of Mesenchymal Stem Cells to not only regenerate tissues of mesenchymal lineages, but also differentiate into cells derived from other embryonic layers, including neurons and epithelia in skin, lung, liver, intestine, kidney, and spleen. ${ }^{77-79}$

Application in Gene transfer: Apart from their ability to self-renew and transdifferentiate, Multipotent Stem Cells have been used as vehicle to introduce exogenous DNA, making them important for tissue regeneration therapies. For example, MSCs infected with an adenovirus vector carrying dominant-negative mutant collagen type I gene have shown to repair the bone in individuals with osteogenesis imperfect, a brittle bone disease. ${ }^{80}$

Prospects: Despite of such vast therapeutic potential of multipotent stem cells, adult MSCs lack telomerase activity. ${ }^{81}$ Therefore, they show definite ex vivo proliferation capability, reach senescence and lose multi-lineage differentiation ability after $34-50$ population doublings in culture. Thus, in order to utilize them to the maximum, it is required to develop methods to prolong the replicative capacity of MSCs with least unfavorable effects on their multi-potency. In this regard, several studies have shown forced ectopic expression of human telomerase reverse transcriptase (hTERT) in postnatal MSCs to extend their life span to more than 260 population doublings, without any impairment in their osteogenic, chondrogenic, adipogenic, neurogenic, and stromal differentiation potential..$^{82,83}$

\section{Discussion}

Intense research on stem cells during the last decades has provided vital information on the processes that govern tissue and organ formation, maintenance, regeneration, and repair after injuries. ${ }^{53,54,59,84-87}$ Moreover, these advancements have offered great promise for developing novel successful stem cell-based medical treatments. $^{73}$ This is based on the possibility of stimulating their ex vivo and in vivo expansion and differentiation into functional progeny that could regenerate the injured tissues/organs in humans..$^{53}$ The field of stem-cell biology has further been catapulted forward by the startling development of reprogramming technology which has created powerful new opportunities for modeling human diseases and offers hope for personalized regenerative cell therapies. ${ }^{88}$

Despite of immense development in the field of stem cell biology, further studies appear to be necessary for clinical therapeutic applications in humans. This includes investigation to optimize the experimental conditions for isolation, expansion, and differentiation of human stem/progenitor cells in vitro, ex vivo, and in vivo. Also, the identification of specific biomarkers to each type of adult stem/ progenitor cells is a precursor for the characterization of their specific physiological functions. In addition, the loss functions of adult stem/ progenitor cells with aging and in specific pathological disorders need to be understood by the elucidation of the complex network of environmental signals that govern the self-renewal and differentiating capacities of these cells. This, in turn, can provide information on the sequence of molecular events that may be associated with each stage of development of a particular disease in humans, hence allowing for personalized therapy designing. In addition, the establishment of engraftment mechanisms of transplanted stem/progenitor cells in contrast to their nonfunctional or malignant counter-part could also lead to new, less toxic therapeutic transplant strategies. ${ }^{73}$

Other obstacles in the way of this type of theory are mostly due to tissue transport, pharmacokinetics, and protein stability, which emphasizes the need to identify all the components of the secretome from which stem cells are extracted for the therapy and to develop novel release and delivery methods. ${ }^{89}$ With the immense flow of research being carried out in the field of stem cell biology, these above mentioned issues will soon be encountered with success, paving way to life-saving therapies.

\section{Conclusion}

Extensive studies and numerous experiments carried out by the scientists have made substantial amount of information available over the years and has unfolded a new chapter of the promising use of stem cells as a therapeutic agent. With the ability to differentiate and proliferate into a spectrum of cells and therefore hold a prominent position in the field of regenerative as well as personalized medicine. Correspondingly, disorders associated with liver, retina, cardiovascular system or neurodegeneration which were previously untreatable due to the limitations exhibited by the immune system 
and also with respect to tissue regeneration have now been cured successfully and effectively. ${ }^{90}$ Furthermore, in accordance with the results of Allison D. Ebert \& Clive N. Svendsen published in Nature, stem cells provide a foothold platform for the process of drug screening as well as drug development; thereby, enabling scientists to study the effects of a drug directly on a human cell line rather than on animal models. ${ }^{1,91,92}$ Consequently, with the aim to repair and replace the damaged organ or tissues, stem cells promise a vivid future and an unparallel approach for the treatment of nearly all pervasive and debilitating human diseases.

\section{Acknowledgements}

Many thanks to all teachers, trainers and friends for imparting enough suggestions and knowledge, which came out as this written review.

\section{Conflict of interest}

The author declares no conflict of interest.

\section{References}

1. Potten CS, Loeffler M. Stem cells: attributes, cycles, spirals, pitfalls and uncertainties. Lessons for and from the crypt. Development. 1990;110(4):1001-1020

2. Brook FA, Gardner RL. The origin and efficient derivation of embryonic stem cells in the mouse. Proc Natl Acad Sci USA. 1997;94(11):57095712 .

3. van de Ven C1, Collins D, Bradley MB, et al. The potential of umbilical cord blood multipotent stem cells for nonhematopoietic tissue and cell regeneration. Exp Hematol. 2007;35(12):1753-1765.

4. Flintoft L. Germ cell poising for totipotency. Nat Rev Genet. 2013;14(11):745.

5. Avasthi S, Shrivastava RN, Singh A, et al. Review article: stem cell: past present and future. IJMU. 2008;3(1):22-30.

6. Hongbao M, Yang Y, Ma M. Totipotent of stem cell. Stem Cell. 2012;3(4):1-5.

7. Reule S, Gupta S. Kidney regeneration and the role of stem cells. Organogenesis. 2011;7(2):135-139.

8. Eckfeldt CE, Mendenhall EM, Flynn CM, et al. Functional analysis of human hematopoietic stem cell gene expression using zebrafish. PLoS Biol. 2005;3(8):e254.

9. Nicholas JS, Hall BV. Experiments on developing rats. II. The development of isolated blastomeres and fused eggs. Journal of Expermental Zoology. 1942;90(3):441-459.

10. Johnson WH, Loskutoff N, Plante Y, et al. Production of four identica calves by the seperation of blastomeres from an in vitro derived 4-cell embryo. Vet Rec. 1995;137(1):15-16.

11. Willadsen SM, Polge C. Attempts to produce monozygotic quadruplets in cattle by blastomere separation. Vet Rec. 1981;108(10):211-213.

12. Tarkowski AK. Experiments on the development of isolated blastomeres of mouse eggs. Nature. 1959;184:1286-1287.

13. Fraser CC, Szilvassy SJ, Eaves CJ, et al. Proliferation of totipotent hematopoietic stem cells in vitro with retention of long term competitive in vivo reconstituting ability. Proc Natl Acad Sci USA. 1992;89(5):19681972 .

14. Mintz B, Anthony K, Litwin S. Monoclonal derivation of mouse myeloid and lymphoid lineages from totipotent hematopoietic stem cells experimentally engrafted in fetal hosts. Proc Natl Acad Sci USA 1984;81(24):7835-7839.
15. Keller GM. In vitro differentiation of embryonic stem cells. Curr Opin Cell Biol. 1995;7(6):862-869.

16. Clarke DL, Johansson CB, Wilbertz J, et al. Generalized Potential of Adult Neural Stem Cells. Science. 2000;288(5471):1660-1663.

17. Ying QL, Nichols J, Evans EP, et al. Changing potency by spontaneous fusion. Nature. 2002;416(6880):545-548.

18. Yu H, Cheng L, Cho KS. The potential of stem cell based therapy for retinal repair. Neural Regen Res. 2014;9(11):1100-1103.

19. Lerou PH, Daley GQ. Therapeutic potential of embryonic stem cells. Blood Rev. 2005;19(6):321-331.

20. Kyba M, Perlingeiro RC, Daley GQ. HoxB4 confers definitive lymphoid-myeloid engraftment potential on embryonic stem cell and yolk sac hematopoietic progenitors. Cell. 2002;109(1):29-37.

21. Kim JH, Auerbach JM, Rodriguez-Gomez JA, et al. Dopamine neurons derived from embryonic stem cells function in an animal model of Parkinson's disease. Nature. 2002;418(6893):50-56.

22. Barberi T, Klivenyi P, Calingasan NY, et al. Neural subtype specification of fertilization and nuclear transfer embryonic stem cells and application in parkinsonian mice. Nat Biotechnol. 2003;21(10):1200-1207.

23. Odorico JS, Kaufman DS, Thomsan JS. Multi lineage differentiation from human embryonic stem cell lines. Stem Cells. 2001;19(3):193-204.

24. Klug MG, Soonpaa MH, Koh GY, et al. Genetically selected cardiomyocytes from differentiating embryonic stem cells from stable intracardiac grafts. J Clin Invest. 1996;98(1):216-224.

25. Müller-Ehmsen J, Kedes LH, Schwinger RHG, et al. Cellular cardiomyoplasty - a novel approach to treat heart disease. Congest Heart Fail. 2002;8(4):220-227.

26. Doetschman TC, Eistetter H, Katz M, et al. The in vitro development of blastocyst-derived embryonic stem cell lines: formation of visceral yolk sac, blood islands and myocardium. J Embryol Exp Morphol. 1985;87:27-45.

27. Yoder MC. Introduction: spatial origin of murine hematopoietic stem cells. Blood. 2001;98(1):3-5.

28. Hochedlinger K, Jaenisch R. Nuclear transplantation, embryonic stem cells, and the potential for cell therapy. $N$ Engl J Med. 2003;349(3):275286.

29. Kiskinis E, Eggan K. Progress toward the clinical application of patient specific pluripotent stem cells. J Clin Invest. 2010;120(1):51-59.

30. Park IH, Lerou PH, Zhao R, et al. Generation of human-induced pluripotent stem cells. Nat Protoc. 2008;3(7):1180-1186.

31. Wernig M, Zhao JP, Pruszak J, et al. Neurons derived from reprogrammed fibroblasts functionally integrate into the fetal brain and improve symptoms of rats with Parkinson's disease. Proc Natl Acad Sci USA. 2008;105(15):5856-5861.

32. Ebert AD, Yu J, Rose FF Jr, et al. Induced pluripotent stem cells from a spinal muscular atrophy patient. Nature. 2009;457(7227):277-280.

33. $\mathrm{Xu} \mathrm{D,} \mathrm{Alipio} \mathrm{Z,} \mathrm{Fink} \mathrm{LM,} \mathrm{et} \mathrm{al.} \mathrm{Phenotypic} \mathrm{correction} \mathrm{of} \mathrm{murine}$ hemophilia A using an iPS cell-based therapy. Proc Natl Acad Sci USA 2009;106(3):808-813.

34. Piekarz RL, Bates SE. Epigenetic modifiers: basic understanding and clinical development. Clin Cancer Res. 2009;15(12):3918-3926.

35. KernS, Eichler H, Stoeve J, et al. Comparative Analysis of Mesenchymal Stem Cells from Bone Marrow, Umbilical Cord Blood, or Adipose Tissue. Stem Cells. 2006;24(5):1294-1301

36. Shi S, Gronthos S, Chen S, et al. Bone formation by human postnatal bone marrow stromal stem cells is enhanced by telomerase expression. Nat Biotechnol. 2002;20(6):587-591. 
37. Horwitz EM, Gordon PL, Koo WK, et al. Isolated allogeneic bone marrow-derived mesenchymal cells engraft and stimulate growth in children with osteogenesis imperfecta: implications for cell therapy of bone. Proc Natl Acad Sci. 2002;99(13):8932-8937.

38. Koc ON, Gerson SL, Cooper BW, et al. Rapid hematopoietic recovery after co infusion of autologous blood stem cells and culture-expanded marrow mesenchymal stem cells in advanced breast cancer patients receiving high-dose chemotherapy. J Clin Oncol. 2000;18(2):307-316.

39. Petite H, Viateau V, Bensaid W, et al. Tissue-engineered bone regeneration. Nat Biotechnol. 2000;18(9):959-963.

40. Kadiyala S, Young RG, Thiede MA, et al. Culture expanded canine mesenchymal stem cells possess osteochondrogenic potential in vivo and in vitro. Cell Transplant. 1997;6(2):125-134.

41. Richards M, Huibregtse BA, Caplan AI, et al. Marrow-derived progenitor cell injections enhance new bone formation during distraction. J Orthop Res. 1999;17(6):900-908.

42. Johnstone B, Yoo JU. Autologous mesenchymal progenitorcells in articular cartilage repair. Clin Orthop Relat Res. 1999;367:S156-S162.

43. Young RG, Butler DL, Weber W, et al. Use of mesenchymal stem cells in a collagen matrix for Achilles tendon repair. J Orthop Res. 1998;16(4):406-413.

44. da Silva Meirelles L, Chagastelles PC, Nardi NB. Mesenchymal stem cells reside in virtually all post-natal organs and tissues. J Cell Sci. 2006;119(11):2204-2213.

45. Zengin E, Chalajour F, Gehling UM, et al. Vascular wall resident progenitor cells:a source for postnatal vasculogenesis. Development. 2006;133(8):1543-1551.

46. Bernardo ME, Emnos JA, Karperien M, et al. Human mesenchymal stem cells derived from bone marrow display a better chondrogenic differentiation compared with other sources. Connect Tissue Res. 2007;48(3):132-140.

47. Chan J, Waddington SN, O'Donoghue K, et al. Widespread distribution and muscle differentiation of human fetal mesenchymal stem cells after intra uterine transplantation in dystrophic mdx mouse. Stem Cells. 2007;25(4):875-884.

48. Yamada Y, Yokoyama S, Wang XD, et al. Cardiac stem cells in brown adipose tissue express CD133 and induce bone marrow non hematopoietic cells to differentiate into cardiomyocytes. Stem Cells. 2007;25(5):1326-1333.

49. Wolbank S, Peterbaur A, Fahrner M, et al. Dose-dependent immune modulatory effect of human stem cells from amniotic membrane:a comparison with human mesenchymal stem cells from adipose tissue. Tissue Eng. 2007;13(6):1173-1183.

50. Price CT, Connolly JF, Carantzas AC, et al. Comparison of bone grafts for posterior spinal fusion in adolescent idiopathic scoliosis. Spine. 2003;28(8):793-798

51. Faundez AA, Taylor S, Kaelin AJ. Instrumented fusion of thoracolumbar fracture with type I mineralized collagen matrix combined with autogenous bone marrow as a bone graft substitute: a four-case report. Eur Spine J. 2006;15(5):630-635.

52. Bryder D, Rossi DJ, Weissman IL. Hematopoietic stem cells: the paradigmatic tissue-specific stem cell. Am J Pathol. 2006;169(2):338346 .

53. Mimeault M, Batra SK. Recent advances on the significance of stem cells in tissue regeneration and cancer therapies. Stem Cells. 2006;24(11):2319-2345.

54. Asahara T, Kawamoto A. Endothelial progenitor cells for postnatal vasculogenesis. Am J Physiol Cell Physiol. 2004;287(3):C572-C579.
55. Friedrich EB, Walenta K, Scharlau J, et al. CD34-/CD133+/VEGFR-2+ endothelial progenitor cell subpopulation with potent vasoregenerative capacities. Circ Res. 2006;98(3):E20-E25.

56. Schatteman GC, Dunnwald M, Jiao C. Biology of bone marrowderived endothelial cell precursors. Am J Physiol Heart Circ Physiol. 2007;292(1):H1-H18.

57. Muller P, Beltrami AP, Cessali D, et al. Myocardial regeneration by endogenous adult progenitor cells. J Mol Cell Cardiol. 2005;39(2):377387.

58. Ourednik J, Ourednik V, Lynch WP, et al. Neural stem cells display an inherent mechanism for rescuing dysfunctional neurons. Nat Biotechnol. 2002;20:1103-1110.

59. Lindvall O, Kokaia Z, Martinez-Serrano A. Stem cell therapy for human neurodegenerative disorders-how to make it work. Nat Med. 2004;10:S42-S50.

60. Chang YC, Shyu WC, Lin SZ, et al. Regenerative therapy for stroke. Cell Transplant. 2007;16(2):171-181.

61. Geraerts M, Krylyshkina O, Debyser Z, et al. Concise review: therapeutic strategies for Parkinson disease based on themodulation of adult neurogenesis. Stem Cells. 2007;25(2):263-270.

62. Trzaska KA, Rameshwar P. Current advances in the treatment of Parkinson's disease with stem cells. Curr Neurovasc Res. 2007;4(2):99109.

63. Ramaswamy S, Shannon KM, Kordower JH. Huntington's disease: pathological mechanisms and therapeutic strategies. Cell Transplant. 2007;16(3):301-312.

64. Lu CZ, Xiao BG, Link H. Neuro protection of G-CSF in cerebral ischemia. Front Biosci. 2007;12:2869-2875.

65. Brazelton TR, Rossi FM, Keshet GI, et al. From marrow to brain: expression of neuronal phenotypes in adult mice. Science. 2000;290(5497):1775-1779.

66. Mezey E, Chandross KJ, Harta G, et al. Turning blood into brain: cells bearing neuronal antigens generated in vivo from bone marrow. Science. 2000;290(5497):1779-1782.

67. Ferrari G, Cusella-De Angelis G, Coletta M, et al. Muscle regeneration by bone marrow-derived myogenic progenitors. Science. 1998;279(5356):1528-1530.

68. Orlic D, kajestura J, Chementi S, et al. Bone marrow cells regenerate infarcted myocardium. Nature. 2001;401(6829):701-705.

69. Jackson KA, Makja SM, Wang H, et al. Regeneration of ischemic cardiac muscle and vascular endothelium by adult stem cells. J Clin Invest. 2001;107(11):1395-1402.

70. Asahara $\mathrm{T}$, Masuda $\mathrm{H}$, Takahashi $\mathrm{T}$, et al. Bone marrow origin of endothelial progenitor cells responsible for postnatal vasculogenesis in physiological and pathological neo vascularization. Circ Res. 1999;85(3):221-228.

71. Lagasse E, Connors H, Al-Dhalimy M, et al. Purified hematopoietic stem cells can differentiate into hepatocytes in vivo. Nat Med. 2000;6(11):1229-1234.

72. Krause DS, Theise ND, Collector MI, et al. Multi-organ, multilineage engraftment by a single bone marrow-derived stem cell. Cell. 2001;105(3):369-377.

73. Mimeault M, Hauke R, Batra SK. Stem Cells: A revolution in therapeutics-recent advances in stem cell biology and their therapeutic applications in regenerative medicine and cancer therapies. Clin Pharmacol Ther. 2007;82(3):252-264. 
74. Kawamoto A, Gwan HC, Iwaquro H, et al. Therapeutic potential of ex vivo expanded endothelial progenitor cells for myocardial ischemia. Circulation. 2001;103(5):634-637.

75. Kocher AA, Schuster MD, Szabolcs MJ, et al. Neo vascularization of ischemic myocardium by human bone-marrow-derived angioblasts prevents cardio myocyto apoptosis, reduces remodeling and improves cardiac function. Nat Med. 2001;7(4):430-436.

76. Kawamoto A, Tkebuchava T, Yamaguchi JI, et al. Intra myocardial transplantation of autologous endothelial progenitor cells for therapeutic neo vascularization of myocardial ischemia. Circulation. 2003;107(3):461-468.

77. Sugaya K. Potential use of stem cells in neuro replacement therapies for neurodegenerative diseases. Int Rev Cytol. 2003;228:1-30.

78. Chapel A, Bertho JM, Bensidhoum M, et al. Mesenchymal stem cells home to injured tissues when co-infused with hematopoieticcells to treat a radiation-induced multi-organ failure syndrome. J Gene Med. 2003;5(12):1028-1038.

79. Deng Y, Guo X, Yuan Q, et al. Efficiency of adeno viral vector mediated CTLA4Ig gene delivery into mesenchymal stem cells. Chin Med J. 2003;116(11):1649-1654.

80. Ortiz LA, Gambelli F, McBride C, et al. Mesenchymal stem cell engraftment in lung is enhanced in response to bleomycin exposure and ameliorates its fibrotic effects. Proc Natl Acad Sci. 2003;100(14):84078411.

81. Chamberlain JR, Schwarze U, Wang PR, et al. Gene targeting in stem cells from individuals with osteogenesis imperfect. Science. 2004;303(5661):1198-1201.
82. Zimmermann S, Voss M, Kaiser S, et al. Lack of telomerase activity inhuman mesenchymal stem cells. Leukemia. 2003;17(6):1146-1149.

83. Simonsen JL, Rosada C, Serakinci N, et al. Telomerase expression extends the proliferative life-span and maintains the osteogenic potential of human bone marrow stromal cells. Nat Biotechnol. 2002;20(6):592596.

84. Bryder D, Rossi DJ, Weissman IL. Hematopoietic stem cells: the paradigmatic tissue-specific stem cell. Am J Pathol. 2006;169(2):338346.

85. Murry CE, Field LJ, Menasche P. Cell-based cardiac repair: reflections at the 10-year point. Circulation. 2005;112(20):3174-3183.

86. Bonner-Weir S, Weir GC. New sources of pancreatic beta-cells. Nat Biotechnol. 2005;23(7):857-861.

87. Barrilleaux B, Phinney DG, Prockop DJ, et al. Review: ex vivo engineering of living tissues with adult stem cells. Tissue Eng. 2006;12(11):3007-3019.

88. Robinton D, Daley G. The promise of induced pluripotent stem cells in research and therapy. Nature. 2012;481(7381):295-305.

89. Flower T, Pulsipher V, Moreno A. A new tool in regenerative medicine: mesenchymal stem cell secretome. J Stem Cell Res Ther. 2015;1(1):1-3.

90. Kuehnle I, Goodell MA. The therapeutic potential of stem cells from adults. BMJ. 2002;325(7360):372-376.

91. Ebert AD, Svendsen CN. Human stem cells and drug screening: opportunities and challenges. Nature Reviews Drug Discovery. 2010;9:367-372.

92. Cressey D. Stem cells take root in drug development. Nature. 2012. 